\title{
Demyelinating syndrome in SLE: review of different disease subtypes and report of a case series
}

\author{
E. Chessa, M. Piga, A. Floris, A. Mathieu, A. Cauli \\ Rheumatology Unit, University Clinic AOU of Cagliari, Italy
}

\section{SUMMARY}

Demyelinating syndrome (DS) is a rare manifestation of systemic lupus erythematosus (SLE) (1\%) with high clinical heterogeneity and potentially severe prognosis. It can represent a diagnostic and therapeutic challenge for clinicians. A recent study described 5 different patterns of demyelinating disease presentation, characterised by specific clinical, laboratory and brain and spine magnetic resonance imaging abnormalities:

1) neuromyelitis optica;

2) neuromyelitis optica spectrum disorders;

3) DS prevalently involving the brain;

4) DS prevalently involving the brainstem;

5) clinically isolated syndrome.

In this review we briefly discuss typical characteristics of each DS presentation in SLE and we describe 5 illustrative clinical cases, one for each subset of DS, considering both diagnostic and therapeutic options.

Key words: Systemic lupus erythematosus; Demyelinating syndrome; Neuropsychiatric lupus; Magnetic resonance.

Reumatismo, 2017; 69 (4): 175-183

\section{INTRODUCTION}

ystemic lupus erythematosus (SLE) is $\checkmark$ a chronic inflammatory disease with an immune-mediated pathogenesis, characterised by a wide range of clinical manifestations (1-3). Neuropsychiatric manifestations in SLE are present in about 50-70\% of patients, and they can involve peripheral, autonomous and central nervous systems (CNS) (4). Neuropsychiatric involvement is associated with a worse prognosis (5). In 1999 the American College of Rheumatology (ACR) proposed criteria for neuropsychiatric SLE (NPSLE) and identified 12 syndromes involving the CNS (6). Demyelinating syndrome (DS), previously defined lupoid sclerosis, which indicated a syndrome similar to multiple sclerosis (MS) in SLE, is one of the rarest and least studied. DS in SLE has a prevalence of $1 \%$ and it has been defined according to the presence of two or more of the following manifesta- tions of demyelinating disease, each occurring at different times (recurring), or one of the following occurring on at least two different occasions (relapsing) (6):

1) multiple discrete areas of damage to white matter (WM) within CNS, causing one or more limbs to become weak with sensory loss;

2) transverse myelopathy (TM);

3) optic neuropathy (ON);

4) diplopia due to isolated nerve palsy or internuclear ophthalmoplegia;

5) brain stem disease with vertigo, vomiting, ataxia, dysarthria or dysphagia;

6) other cranial nerve palsies.

A recent review has described 5 different subsets of DS in SLE on the basis of clinical, serologic and imaging criteria (7):

1) neuromyelitis optica (NMO);

2) neuromyelitis optica spectrum disorders (NMOSD);

3) demyelinating syndrome prominently involving the brain (DSB);

\section{Conference} presentation: paper presented at SIR Congress, 2016.
Corresponding author Elisabetta Chessa

Struttura Complessa di Reumatologia, Policlinico Universitario,

AOU di Cagliari SS 554 09042 Monserrato - Cagliari, Italy E-mail: elis.chessa@gmail.com 
4) demyelinating syndrome prominently involving the brainstem (DSBS);

5) clinically isolated syndrome (CIS). CIS, which accounts for the first episode of MS in $85 \%$ of cases (8), is defined as a demyelinating manifestation isolated in time. The lack of recurring and relapsing features excludes CIS from the 1999 ACR classification for NPSLE. However, CIS has been described as representing one third of DS secondary to SLE and for this reason it should be included in NPSLE definition (7).

The aim of this study is to describe the 5 subsets of DS as NPSLE manifestation through the analysis of illustrative clinical cases from the SLE cohort of patients followed-up at the Rheumatology Unit of the University Hospital of Cagliari (9).

\section{NMO - NEUROMYELITIS OPTICA}

NMO is characterised by optic neuritis $(\mathrm{ON})$ and myelitis and it was considered for long time as a variant of MS known as Devic's syndrome. In 1999, Wingerchuck et al. (10) defined some of the distinctive characteristics of NMO: relapsing course, evidence of a contiguous spinal cord lesion involving 3 or more segments in length (LETM, longitudinally extensive transverse myelitis), pleocytosis and absence of oligoclonal bands $(\mathrm{OB})$ in the cerebrospinal fluid (CSF). Later, the identification of a specific serum autoantibody marker, targeting water channel Aquaporin-4 (NMO$\mathrm{IgG}$ ) on astrocytes, contributed to defining $\mathrm{NMO}$ as an isolated disorder. According to the 2006 classification criteria for NMO $(11,12)$, its diagnosis is supported by the presence of optic neuritis and acute myelitis in association with at least two of the following:

1) contiguous spinal cord MRI lesion extending over 3 vertebral segments (LETM);

2) onset brain MRI not meeting the diagnostic criteria for MS;

3) NMO-IgG seropositivity status.

In a recent review (7), $13.5 \%$ of DS in SLE satisfied the 2006 criteria for NMO. LETM predominantly involved the WM (78.6\%) with spasticity, hyperreflexia and urinary incontinence. Positivity for NMO-IgG was described in $62.5 \%$ of patients, while brain MRI lesions were showed in hypothalamic, periventricular and corpus callosum WM, or in the brainstem, in more than a half of NMO patients $(57.1 \%)$. CSF analysis generally showed pleocytosis and hyperprotidorrachia, but $\mathrm{OB}$ were detected only in $22.2 \%$ of patients. NMO was the DS subtype associated with the worst prognosis, with a high frequency of relapses, clinical worsening and death in $50 \%$ of cases, despite treatment (7).

\section{Case report NMO}

In 1996, a 32 year old female reported sudden visual loss in her right eye and she was diagnosed with ON. A few days later, she developed lower limbs weakness with spasticity, hyperreflexia and paraesthesia. She also had malar rash, class III glomerulonephritis, ANA 1:640 homogeneous, anti-dsDNA (176 UI/l using RIA; NV $<7)$ and low levels of serum C3 $43 \mathrm{mg} / \mathrm{dl}$ (NV 83-177) and C4 $12 \mathrm{mg} / \mathrm{dl}$ (NV 20-47) complement fractions. She was positively tested for anti-Sm, anti-RNP, and anti-Ro/ SSA. Lupus anticoagulant (LA), anti-Cardiolipin (aCL) IgG and IgM were negative. Spinal cord MRI showed a C2-C6 LETM (Figure 1), while CSF analysis revealed IgG $16.2 \mathrm{mg} / \mathrm{dl}(\mathrm{NV}<3.5)$ with intrathecal OB synthesis. Infections were ruled out. Thus, she was diagnosed with SLE and DS (NMO), treated with $500 \mathrm{mg}$ pulses of methylprednisolone (MPRE) for three consecutive days and monthly cyclophosphamide (CYC) pulses $\left(750 \mathrm{mg} / \mathrm{m}^{2}\right.$ body surface) for 6 months. She experienced motor and sensory clinical improvement, but the blindness in her right eye did not recover. Azathioprine $100 \mathrm{mg}$ and low dose prednisolone (PRE) were given as maintenance treatment with long-term clinical remission; nevertheless she was burdened with residual neurologic damage.

In 2005, while still on maintenance treatment, she had a relapse with rapid progression to hemiparesis, new appearance of myoclonus and urinary incontinence. She 
had no other manifestations of SLE activity but low C3 $28 \mathrm{mg} / \mathrm{dl}$ (NV 83-177) and C4 $2 \mathrm{mg} / \mathrm{dl}$ (NV 20-47). Spinal cord MRI was normal. Brain MRI showed large confluent inflammatory lesions, hyperintense in T2 and FLAIR sequences, without contrast enhancement, involving the periventricular $\mathrm{WM}$, semioval centres, corona radiata and internal capsule secondary to a NMO relapse. Search for NMO-IgG was not performed. She was treated with a new cycle of MPRE pulses and CYC for 6 months without neurologic improvement.

\section{NMOSD - NEUROMYELITIS OPTICA SPECTRUM DISORDERS}

Overall, $42.9 \%$ of DS in SLE satisfy the 2007 criteria for NMOSD (7), presenting at least one of the following manifestations, with or without brain involvement (13):

1) recurrent events of longitudinally extensive myelitis ( $\geq 3$ vertebral segment spinal cord lesion seen on MRI);

2) optic neuritis, recurrent or simultaneous bilateral;

3) optic neuritis associated with myelitis (not longitudinally extensive).

Birnbaum et al. (14) described two distinct syndromes with spinal cord involvement in SLE: one that prominently involves $\mathrm{WM}$ and one mainly affecting grey matter (GM). Patients with prevailing WM dysfunction show spasticity, hyperreflexia and a relapsing pattern, generally meeting the criteria for NMO and more likely to have LA. GM involvement is generally associated with clinical prodromes of fever and urinary retention (often causing diagnostic delay), flaccidity and hyporeflexia. These patients are more likely to have irreversible paraplegia despite presenting with a monophasic course (14).

The most frequent manifestation of NMOSD in SLE is LETM (57.1\%), with WM or GM findings, mainly involving the cervical and thoracic spinal cord segments with MRI contrast enhancement in one third of cases (7). Less frequently, NMOSD presents as $\mathrm{ON}$ associated with transverse myelitis $(28.6 \%)$ or as isolated recurrent/

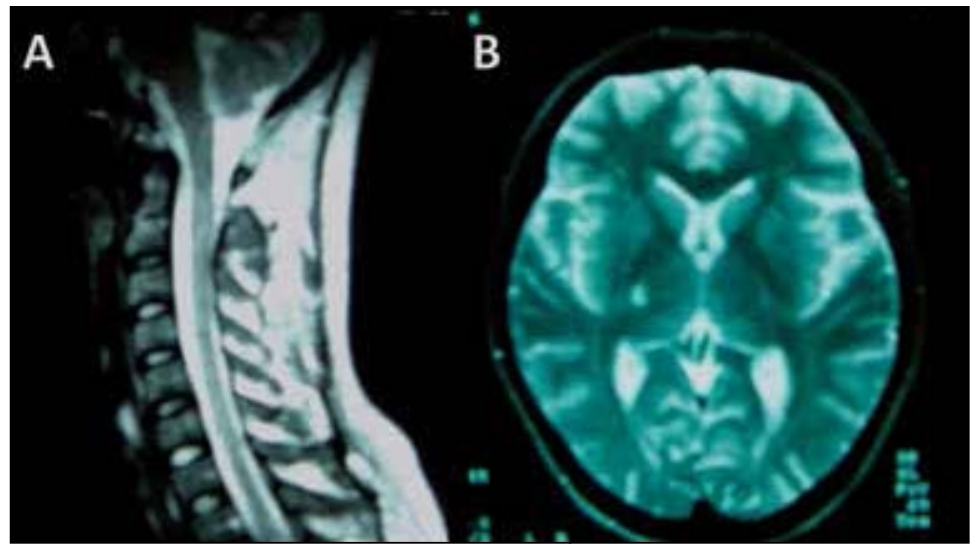

Figure 1 - A) Sagittal image on T2-weighted sequences of cervical spine MRI showing LETM from C2 to C6 without contrast-enhancement. B) Transversal image of brain MRI showing a small hyperintense lesion on T2-weighted sequences on the internal capsule, without contrast-enhancement.

bilateral ON (14.3\%), which is associated with a better prognosis. Hyperprotidorrachia and pleocytosis were the most frequent CSF findings in NMOSD, whereas OB $(<10 \%)$ were seldom observed. NMOIgG were detected in $10 \%$ of cases (7).

The variety of NMOSD clinical presentations mirrored the variability of prognosis, which ranged from complete or partial resolution in half cases, to stable course despite treatment in $25 \%$ of cases, to worsening and death in the remaining 25\% (7).

\section{Case report (NMOSD)}

On June 1994, a 44 year old woman was admitted to the Neurology Department for progressive gait impairment reaching paraplegia with areflexia, urinary retention and hypoesthesia in lower limbs preceded by nausea and vomiting. Two months earlier, she had experienced the same symptoms followed by spontaneous remission. On physical examination she exhibited malar rash and she described having suffered from photosensitivity and oral ulcers, without xerostomia or xerophthalmia. Laboratory analysis showed ANA 1:160 speckled, anti-Ro/SSA and anti-La/SSB positivity, C3 $77 \mathrm{mg} / \mathrm{dl}$ (NV 83-177), C4 1 $\mathrm{mg} / \mathrm{dl}$ (NV 20-47), whilst anti-dsDNA and antiphospholipid antibodies (LA and aCL) were negative. Spinal cord MRI showed diffused T2-weighted hyperintensity ex- 
tending from $\mathrm{C} 1$ to T12, with cord swelling and without contrast enhancement. On brain MRI, T2 hyperintense lesions in the left parietal lobe, right parietal-temporaloccipital lobes and corpus callosum, with no contrast enhancement, were found. CSF analysis revealed hyperprotidorrachia, without $\mathrm{OB}$ or evidence of infections. After consultation with a rheumatologist she was diagnosed as having SLE with encephalomyelitis (NMOSD). She was treated with $1 \mathrm{~g}$ MPRE pulses for 5 consecutive days, followed by high doses of PRE $(1 \mathrm{mg} / \mathrm{kg} /$ day) with scheduled tapering, and physical rehabilitation. Six months later she only had slight lower limbs weakness with minimal hyperreflexia. MRI showed reduction in number and size of brain lesions, but a

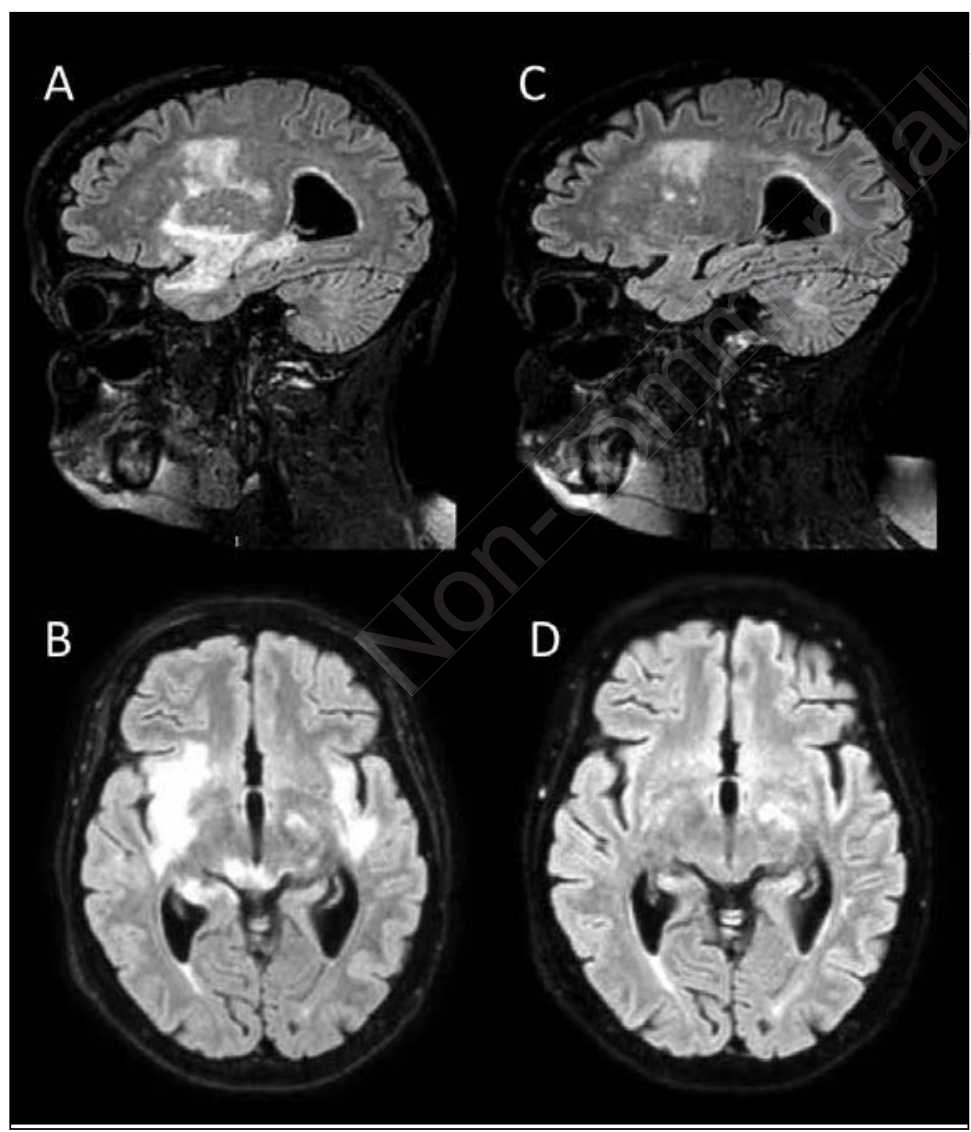

Figure 2 - Brain MRI, sagittal (A) and transversal (B) sections, showing extensive and confluent hyperintense T2-lesions in parietal-temporal lobes bilaterally, particularly involving claustrum, external capsule and midbrain. Initial signs of cortical atrophy. Six months later, at follow up, the same sections showed reduction in size of parietal-temporal lesions (C) and disappearance of brainstem lesions (D). still lasting hyperintense central-medullar band. A further corticosteroid tapering was planned with PRE $25 \mathrm{mg}$ on alternate days. One year later she was completely recovered.

\section{DSB - DS PROMINENTLY INVOLVING THE BRAIN}

The DSB subset defines those DS in SLE $(5.7 \%)$ with predominant brain and cerebellum involvement that do not fulfil NMO or NMOSD criteria (7). Clinical manifestations are extremely pleotropic and related to the size and site of cerebral or cerebellar lesions, and may include nystagmus, diplopia, cranial nerve palsies, ataxia, cognitive dysfunction and acute confusional state extending to coma (7). Other neurological manifestations may be observed, especially headache, seizures, aseptic meningitis and psychosis. CSF analysis frequently shows pleocytosis and hyperprotidorrachia without OB.

\section{Case report (DSB)}

A 49-year old woman, who had been suffering from SLE for 15 years because of polyarthritis, photosensitivity, malar rash, leukopenia, ANA 1:320 homogeneous, anti-Ro/SSA positive and low serum complement fractions, was admitted to our department on February 2016 for neurological disturbances and worsening of renal function. On clinical examination she had severe cognitive dysfunction, dysphagia, dysarthria, ataxia, bilateral VI and left VII cranial nerve palsies, lower limbs hyperreflexia and weakness with impaired left foot dorsiflexion. She also had secondary Sjögren's syndrome and Jaccoud's arthropathy. Laboratory examinations revealed ANA positivity (1:160 homogeneous), anti-Ro/SSA positivity, C3 $72 \mathrm{mg} / \mathrm{dl}$ (NV 90-180), C4 $11 \mathrm{mg} / \mathrm{dl}$ (NV 10-40), serum creatinine $2 \mathrm{mg} / \mathrm{dl}$, glomerular filtration rate $31 \mathrm{ml} / \mathrm{min}$, proteinuria $0.6 \mathrm{~g} / 24 \mathrm{~h}$ but no active urinary sediment. Brain MRI displayed WM extensive and confluent hyperintense lesions on T2 and FLAIR sequences involving the temporal lobes bilaterally (Figure 2), pons and midbrain with 
vasogenic oedema in DWI sequences. Basal ganglia calcifications and mild cortical atrophy were also shown. CSF analysis disclosed hyperprotidorrachia $(46.8 \mathrm{mg} / \mathrm{dl})$, pleocytosis (10 lymphocytes), without OB and with no evidence of infectious diseases (e.g. JCV). Electromyography showed sensory and motor conduction abnormalities on the left common peroneal nerve and both sural nerves. She was diagnosed with DSB in SLE, multiplex mononeuritis and Fahr's disease. Prompt treatment with MPRE 500 $\mathrm{mg}$ pulses for 3 consecutive days, CYC $500 \mathrm{mg}$ single pulse and rituximab $1 \mathrm{~g}$ repeated after 2 weeks were administered. Maintenance therapy with mycophenolate mofetil (MMF) $1 \mathrm{~g} /$ day and progressively tapered PRE $50 \mathrm{mg} /$ day were prescribed. Six months later she showed neuropsychiatric and brain MRI lesions improvement, with complete disappearance of brainstem lesions and vasogenic oedema.

\section{DSBS - DS PROMINENTLY INVOLVING THE BRAINSTEM}

DSBS includes those DS with prominent brainstem involvement $(6 \%)$ that do not fulfil international criteria for $\mathrm{NMO}$ or NMOSD (7). The most frequently described symptoms are diplopia (100\%), nystagmus (71.4\%), and dysarthria (57.4\%), whereas ataxia, dysphagia, dizziness and hiccups were less frequent. The pons, midbrain and cerebellar peduncles are the most involved sites on MRI (7). CSF analysis is usually normal (7).

\section{Case report (DSBS)}

We report on a 40 year old woman, admitted to our unit in 2013 for polyarthritis, pleuro-pericarditis and neurological disturbances characterised by mono-lateral nystagmus, vertigo, dysphagia and dysarthria, which exacerbated during her period. Laboratory examinations revealed ANA 1:160 homogeneous, anti-Ro/SSA positivity, LA positivity, aCL IgG 34 GPL (NV<12 GPL) and anti- 32 -glicoprotein-I IgG 45 GPL $(\mathrm{NV}<12 \mathrm{GPL})$. She was on warfarin (INR target 2.5 to 3 ) because of antiphospholipid syndrome (APS) diagnosed in 2007 for deep venous thrombosis and LA positivity in more than 2 occasions. Brain MRI revealed T2 and FLAIR hyperintense lesions in the medulla oblongata, pons, midbrain and septum-pellucidum interface, associated with leptomeningeal contrast enhancement on brainstem surface and interpeduncular cistern. MRI spectroscopy showed increased choline peaks without $\mathrm{N}$ acetil-aspartate depletion, suggestive of inflammation and demyelination. CSF analysis showed a $0.72 \mathrm{IgG}$ index $(\mathrm{NV}<0.7)$, no $\mathrm{OB}$ and no microbiological abnormalities. She was diagnosed with DSBS in SLE and APS, and treated with monthly CYC pulses (750 $\mathrm{mg} / \mathrm{m}^{2}$ body surface) and high glucocorticoid doses for 6 months, followed by maintenance therapy with MMF 2 g/day and progressive tapering of corticosteroid dosage. After one year of follow-up she had complete remission of neurological disturbances and disappearance of MRI lesions.

\section{CIS - CLINICALLY ISOLATED SYNDROME}

CIS is defined as the first clinical demyelinating event, lasting at least $24 \mathrm{~h}$, with evidence of discrete neurologic lesions in CNS. CIS must be isolated in time (one manifestation, e.g. only $\mathrm{ON}$ ), but it may or may not be isolated in space (more contemporary manifestations, for example $\mathrm{ON}$ and myelitis) (7). CIS could mimic each of the previously described 4 different subtypes of DS presenting more frequently $(62.1 \%)$ as LETM (CIS-NMOSD), in $31 \%$ as brain involvement (CIS-Brain) and in $6.9 \%$ as brainstem involvement (CIS-Brainstem) (7). Isolated $\mathrm{ON}$ is not considered as CIS because it is classified as cranial neuropathy according to the 1999 ACR nomenclature (6). None of the cases reported in literature showed OB or NMO-IgG antibodies. This subset of DS in SLE has the most favourable outcome, with clinical improvement in more than $90 \%$ of cases (7).

\section{Case report (CIS)}

A 43-year old woman was admitted to our department in September 2008 because of non-erosive arthritis, cutaneous discoid 
lupus, photosensitivity and associated left hemiparesis with hypoesthesia in the left part of her body. On laboratory examinations she had ANA 1:320 speckled, antiRo/SSA and LA positivity with low C3 88 $\mathrm{mg} / \mathrm{dl}(\mathrm{NV} 112-220)$ and $\mathrm{C} 411 \mathrm{mg} / \mathrm{dl}$ (NV 12-60) complement fractions. Spinal cord MRI highlighted T2-hyperintense lesions extending to C2-C5 and D1-D9 metameres, with normal brain imaging. CSF analysis was not performed. Diagnosed as SLE with LETM, she was treated with 3 MPRE pulses $(500 \mathrm{mg} /$ day), followed by daily PRE $50 \mathrm{mg}$ and daily CYC $50 \mathrm{mg}$ for 20 days every month. Six months later, she showed recovery of strength but persistence of left

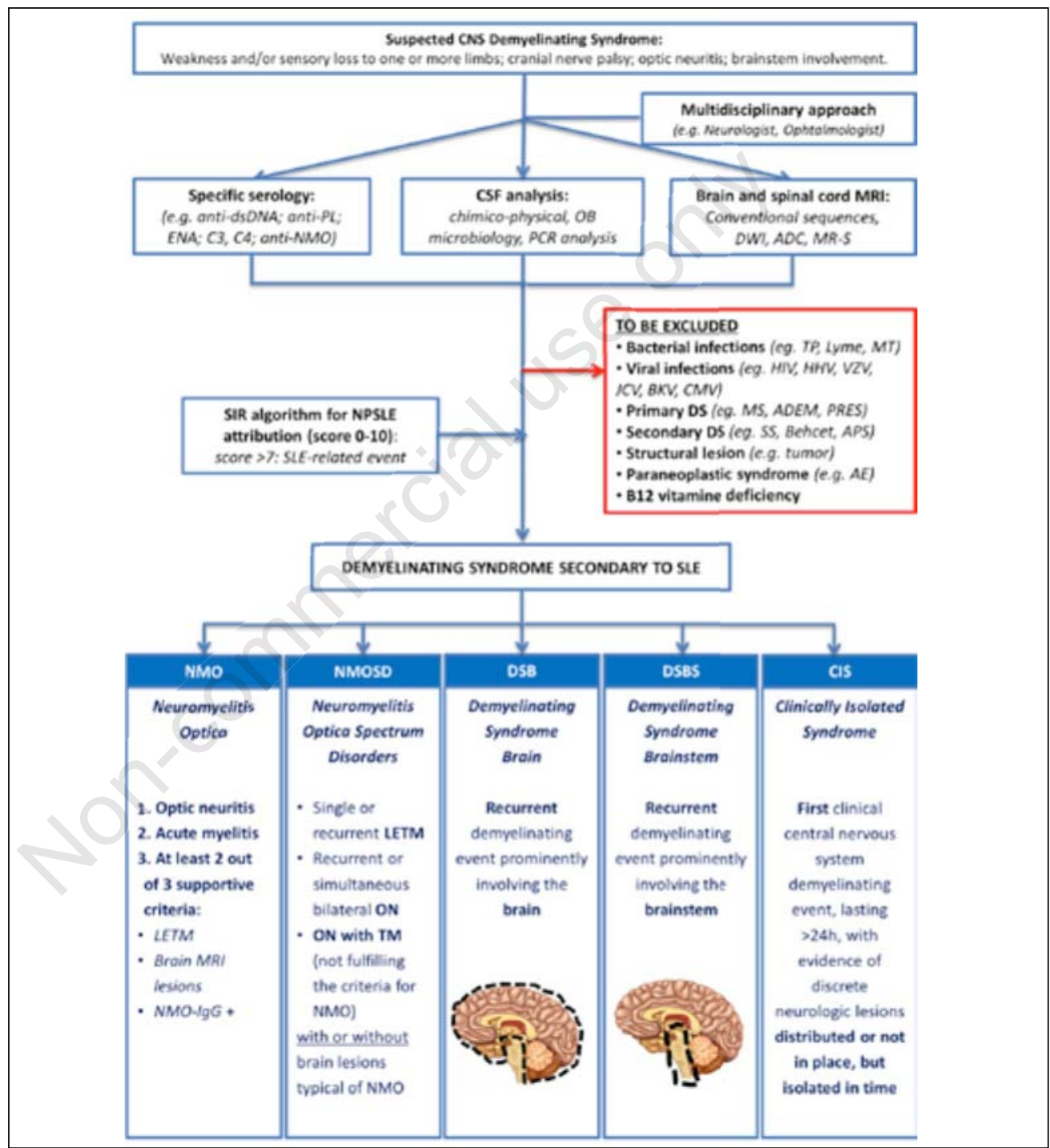

Figure 3 - Algorithm for the approach to diagnosis and attribution of demyelinating syndrome to neuropsychiatric systemic lupus erythematosus. ADC, apparent diffusion coefficient; ADEM, acute demyelinating encephalomyelitis; AE, autoimmune encephalitis; anti-PL, anti-phospholipids; CNS, central nervous system; CSF, cerebrospinal; DS, demyelinating syndrome; DWI, diffusion weighted imaging; LETM, longitudinally extensive transverse myelitis; MRI, magnetic resonance imaging; MR-S, magnetic resonance-spectroscopy; MS, multiple sclerosis; MT, Mycobacterium tuberculosis; NPSLE, neuropsychiatric systemic lupus erythematosus; OB, oligoclonal bands; ON, optic neuritis; PCR, polymerase chain reaction; PRES, posterior reversible encephalopathy syndrome; TP, Treponema pallidum. 
arm paraesthesia, with disappearance of MRI demyelinating lesions.

\section{DISCUSSION AND CONCLUSIONS}

DS secondary to SLE is rare and can represent a diagnostic challenge for clinicians. In the reported cases, the diagnosis of DS secondary to SLE was made only after a careful exclusion process (Figure 3 ), which included primary demyelinating diseases, infectious diseases, neoplastic and paraneoplastic syndromes (15-18). For an accurate differential diagnosis, medical history (e.g. the presence of infections or vaccinations may suggest acute demyelinating encephalomyelitis), and a full clinical examination (e.g. high blood pressure may be found in posterior reversible encephalopathy syndrome) are essential. In 40\% of cases, DS was the onset manifestation of SLE. Searching for specific clinical and serological markers such as anti-dsDNA, specific ENAs and anti-phospholipids is recommended at the first demyelinating event $(7,19)$. In patients with LETM and/ or ON, dosing NMO-IgG could be helpful to identify those DS subtypes with higher risk of relapse and worse prognosis (NMO, NMOSD). While suspecting DS, CSF analysis is mandatory to exclude infections through Gram staining, culture and molecular biology tests aimed at identifying bacteria (e.g., Mycobacteria) and viruses (JCV, BKV, VZV, CMV, HHV) (20). Moreover, CSF analysis in patients with DS secondary to SLE could recognise inflammatory changes, like pleocytosis and hyperprotidorrachia, and also OB $(5 \%$ in literature) even if more rarely than in MS (21). NPSLE and MS have similar pathogenic and clinical characteristics $(22,23)$, but the 2010 revised McDonald's criteria (24) and the recently developed algorithm for attribution of neuropsychiatric events to SLE (25) could be helpful in distinguishing between DS associated with SLE and MS (Table I).

The most accurate imaging procedure to identify demyelinating lesions is MRI (14, 26, 27). Myelopathy is the most frequent finding, being detected in $70 \%$ of DS in SLE, more frequently in the cervical region and less often in thoracic metameres. Brain MRI is characterised by atypical, large and usually confluent T2-weighted and FLAIR inflammatory hyperintense lesions involving periventricular and deep brain WM (e.g., semioval centrum, corona radiata, internal capsule, cerebral peduncles), corpus callosum and brainstem $(7,14,28)$. Contrast enhancement is rare, but if present it is usually detected in spinal cord lesions, in optic nerves or may be leptomeningeal as a marker of small vessels vasculitis (7). In patients with SLE presenting with DS and inflammatory type lesions on brain MRI, especially if spinal cord and optic nerves involvement are absent, progressive multifocal leukoencephalopathy must be suspected as demyelinating disorder secondary to $\mathrm{JC}$ and $\mathrm{BK}$ polyomaviruses infection $(29,30)$. Other findings are cortical atrophy and basal ganglia calcifications, also called Fahr's disease. The latter has been reported in SLE as a result of chronic inflammatory brain damage and may present as Parkinson-like movement disorders, cognitive deficit or dementia $(31,32)$. In evaluating CNS inflammatory disease activity MRI spectroscopy can be helpful to mark neuronal or axonal damage (reduction in $\mathrm{N}$-acetylaspartate peak) or increased metabolic activity (increased choline peak), as observed in inflammatory conditions.

Table I - Favouring and confounding factors for attribution of demyelinating syndrome to SLE according to the algorithm defined by SIR study group on NPSLE.

\begin{tabular}{|l|l|}
\hline Favouring factors & Confounding factors \\
\hline a) SLEDAI >6 & a) Anatomical lesions (e.g., artero-venous malformations, tumors) \\
b) Antiphospholipid antibodies & b) Familial disorders (e.g., ataxia, leukodystrophy, hereditary paraplegia) \\
c) Less than 4 oligoclonal bands & c) Sarcoidosis, Behçet syndrome, vasculitis \\
on CSF analysis & d) Multiple sclerosis \\
\hline
\end{tabular}


According to international recommendations for treatment of severe NPSLE (33), in our cases the induction therapy was based on high oral dose (PRE $0.5-1 \mathrm{mg} / \mathrm{kg} /$ day) or pulsed corticosteroids (MPRE 500 $\mathrm{mg} /$ day for 3-5 days) followed by oral or pulsed CYC (750 $\mathrm{m}^{2}$ body surface) and in a single case rituximab was also administered. Given the need to reduce the daily and cumulative dose of corticosteroids and considering the high risk of relapse, maintenance therapy with MMF (2 g/day) or azathioprine $(1.5-2.5 \mathrm{mg} / \mathrm{kg} /$ day $)$ was prescribed. Moreover, a rigorous and preventive therapeutic approach should be addressed to those general risk factors associated with NPSLE development (34). The outcome of DS mirrored literature data, showing that the worst prognosis belongs to NMO whereas a more favourable prognosis is associated with other DS subsets, and especially CIS (7).

In conclusion, 5 different subtypes of DS in SLE exist and their early identification could be crucial for setting up an accurate differential diagnosis and a prompt treatment.

\section{REFERENCES}

1. Cervera R, Khamashta MA, Font J, et al. Morbidity and mortality in systemic lupus erythematosus during a10-year period: a comparison of early and late manifestations in a cohort of 1,000 patients. Medicine (Baltimore). 2003; 82: 299-308.

2. Sebastiani GD, Prevete I, Piga M, et al. Early lupus project-a multicenter Italian study on systemic lupus erythematosus of recent onset. Lupus. 2015; 24: 1276-82.

3. Piga M, Vacca A, Porru G, et al. Liver involvement in systemic lupus erythematosus: incidence, clinical course and outcome of lupus hepatitis. Clin Exp Rheumatol. 2010; 28: 504-10.

4. Piga M, Mathieu A. Managing CNS involvement in systemic lupus erythematosus. Int $\mathbf{J}$ Clin Rheumatol. 2011; 6: 547-67.

5. Zirkzee EJ, Huizinga TW, Bollen EL, et al. Mortality in neuropsychiatric systemic lupus erythematosus (NPSLE). Lupus. 2014; 23: 31-8.

6. The American College of Rheumatology nomenclature and case definitions for neuropsychiatric lupus syndromes. Arthritis Rheum. 1999; 42: 599-608.
7. Piga M, Chessa E, Peltz MT, et al. Demyelinating syndrome in SLE encompasses different subtypes: Do we need new classification criteria? Pooled results from systematic literature review and monocentric cohort analysis. Autoimmun Rev. 2017; 16: 244-52.

8. Miller D, Barkhof F, Montalban X, et al. Clinically isolated syndromes suggestive of multiple sclerosis, part I: natural history, pathogenesis, diagnosis, and prognosis. Lancet Neurol. 2005; 4: 281-8.

9. Piga M, Casula L, Perra D, et al. Populationbased analysis of hospitalizations in a WestEuropean region revealed major changes in hospital utilization for patients with systemic lupus erythematosus over the period 20012012. Lupus. 2016; 25: 28-37.

10. Wingerchuk DM, Hogancamp WF, O'Brien PC, Weinshenker BG. The clinical course of neuromyelitis optica (Devic's syndrome). Neurology. 1999; 53: 1107-14.

11. Papadopoulos MC, Verkman AS. Aquaporin 4 and neuromyelitis optica. Lancet Neurol. 2012; 11: 535-44.

12. Wingerchuk DM, Lennon VA, Pittock SJ, et al. Revised diagnostic criteria for neuromyelitis optica. Neurology. 2006; 66: 1485-9.

13. Wingerchuk DM, Lennon VA, Lucchinetti CF, et al. The spectrum of neuromyelitis optica. Lancet Neurol. 2007; 6: 805-15.

14. Birnbaum J, Petri M, Thompson R, et al. Distinct subtypes ofmyelitis in systemic lupus erythematosus. Arthritis Rheum. 2009; 60: 3378-87.

15. Theodoridou A, Settas L. Demyelination in rheumatic diseases. J Neurol Neurosurg Psychiatry. 2006; 77: 290-5.

16. Love S. Demyelinating diseases. J Clin Pathol. 2006; 59: 1151-9.

17. Karussis D. The diagnosis of multiple sclerosis and the various related demyelinating syndromes: a critical review. J Autoimmun. 2014; 48-49: 134-42.

18. Magro Checa C, Cohen D, Bollen ELEM, et al. Demyelinating disease in SLE: is it multiple sclerosis or lupus? Best Pract Res Clin Rheumatol. 2013; 27: 405-24.

19. Floris A, Piga M, Cauli A, Mathieu A. Predictors of flares in systemic lupus erythematosus: preventive therapeutic intervention based on serial anti-dsDNA antibodies assessment. Analysis of a monocentric cohort and literature review. Autoimmun Rev. 2016; 15: 656-63.

20. West SG, Emlen W, Wener MH, Kotzin BL. Neuropsychiatric lupus erythematosus: a 10-year prospective study on the value of diagnostic tests. Am J Med. 1996; 99: 153-63.

21. Mclean BN, Miller D, Thompson EJ. Oligoclonal banding of $\mathrm{IgG}$ in CSF, blood-brain barrier function, and MRI findings in patients 
with sarcoidosis, systemic lupus erythematosus, and Behcet's disease involving the nervous system. J Neurol Neurosurg Psychiat. 1995; 58: 548-54.

22. Steri M, Orrù V, Idda ML, et al. Overexpression of the cytokine BAFF and autoimmunity risk. N Engl J Med. 2017; 376: 1615-26.

23. Fanouriakis A, Mastorodemos V, Pamfil C, et al. Coexistence of systemic lupus erythematosus and multiple sclerosis: prevalence, clinical characteristics, and natural history. Semin Arthritis Rheum. 2014; 43: 751-8.

24. Polman CH, Reingold SC, Banwell B, et al. Diagnostic criteria for multiple sclerosis: 2010 revisions to the McDonald criteria. Ann Neurol. 2011; 69: 292-302.

25. Bortoluzzi A, Scirè CA, Bombardieri $S$, et al. Development and validation of a new algorithm for attribution of neuropsychiatric events in systemic lupus erythematosus. Rheumatology (Oxford). 2015; 54: 891-8.

26. Luyendijk J, Steens SCA, Ouwendijk WJN, et al. Neuropsychiatric systemic lupus erythematosus: lessons learned from magnetic resonance imaging. Arthritis Rheum. 2011; 63: 722-32.

27. Piga M, Peltz MT, Montaldo C, et al. Twenty-year brain magnetic resonance imaging follow-up study in systemic lupus erythematosus: factors associated with accrual of damage and central nervous system involvement. Autoimmun Rev. 2015; 14: 510-6.

28. Govoni M, Bortoluzzi A, Padovan M, et al. The diagnosis and clinical management of the neuropsychiatric manifestations of lupus. J Autoimmun. 2016; 74: 41-72.

29. Calabrese LH, Molloy ES, Huang D, Ransohoff RM. Progressive multifocal leukoencephalopathy in rheumatic diseases: evolving clinical and pathologic patterns of disease. Arthritis Rheum. 2007; 56: 2116-28.

30. Daveson KL, Ong CW, Bowden S, et al. BK virus-associated progressive multifocal leukoencephalopathy. Med J Aust. 2013; 198: 216-8.

31. Appenzeller S, Rondina JM, Li LM, et al. Cerebral and corpus callosum atrophy in systemic lupus erythematosus. Arthritis Rheum. 2005; 52: 2783-9.

32. Sibbitt Jr WL, Brooks WM, Kornfeld M, et al. Magnetic resonance imaging and brain histopathology in neuropsychiatric systemic lupus erythematosus. Semin Arthritis Rheum. 2010; 40: 32-52.

33. Bertsias G, Ioannidis JPA, Boletis J, et al. EULAR recommendations for the management of systemic lupus erythematosus. Report of a Task Force of the EULAR Standing Committee for International Clinical Studies Including Therapeutics. Ann Rheum Dis. 2008; 67: 195-205.

34. Govoni M, Bombardieri S, Bortoluzzi A, et al. Factors and comorbidities associated with first central nervous system neuropsychiatric event in systemic lupus erythematosus: does a risk profile exist? A large multicenter retrospective cross sectional study on 959 Italian patients. Rheumatology (Oxford). 2012; 51: 157-68. 